\title{
Demonstration of Entanglement of Electrostatically Coupled Singlet-Triplet Qubits
}

\author{
M. D. Shulman ${ }^{1 \dagger}$, O. E. Dial ${ }^{1 \dagger}$, S. P. Harvey ${ }^{1}$, H. Bluhm $^{1 *}$, V. Umansky ${ }^{2}$, and A. Yacoby ${ }^{1}$ \\ ${ }^{1}$ Department of Physics, Harvard University, Cambridge, MA, 02138, USA \\ ${ }^{2}$ Braun Center for Submicron Research, Department of Condensed Matter Physics, Weizmann Institude of \\ Science, Rehovot 76100 Israel \\ ${ }^{*}$ Current Address: 2nd Institute of Physics C, RWTH Aachen University, 52074 Aachen, Germany \\ ${ }^{\dagger}$ These authors contributed equally to this work.
}

\begin{abstract}
Quantum computers have the potential to solve certain interesting problems significantly faster than classical computers. To exploit the power of a quantum computation it is necessary to perform interqubit operations and generate entangled states. Spin qubits are a promising candidate for implementing a quantum processor due to their potential for scalability and miniaturization. However, their weak interactions with the environment, which leads to their long coherence times, makes inter-qubit operations challenging. We perform a controlled two-qubit operation between singlet-triplet qubits using a dynamically decoupled sequence that maintains the two-qubit coupling while decoupling each qubit from its fluctuating environment. Using state tomography we measure the full density matrix of the system and determine the concurrence and the fidelity of the generated state, providing proof of entanglement.
\end{abstract}

Singlet-triplet ( $S$ - $T_{0}$ ) qubits, a particular realization of spin qubits[1-7], store quantum information in the joint spin state of two electrons[8-10]. The basis states for the $S$ - $T_{0}$ qubit can be constructed from the eigenstates of a single electron spin, $|\uparrow\rangle$ and $|\downarrow\rangle$. We choose $|S\rangle=\frac{1}{\sqrt{2}}(|\uparrow \downarrow\rangle-|\downarrow \uparrow\rangle)$ and $\left|T_{0}\right\rangle=\frac{1}{\sqrt{2}}(|\uparrow \downarrow\rangle+|\downarrow \uparrow\rangle)$ for the logical subspace of the $S-T_{0}$ qubit because these states are insensitive to uniform fluctuations in the magnetic field. The qubit can then be described as a two level system with a representation on a Bloch sphere shown in Fig. 1a. 
Universal quantum control is achieved using two physically distinct operations that drive rotations around the $\mathrm{x}$ and $\mathrm{z}$-axes of the Bloch sphere [11]. Rotations around the $\mathrm{z}$-axis of the Bloch sphere are driven by the exchange splitting, $J$, between $|S\rangle$ and $\left|T_{0}\right\rangle$, and rotations around the x-axis are driven by a magnetic field gradient, $\Delta B_{z}$ between the electrons.

We implement the $S$ - $T_{0}$ qubit by confining two electrons to a double quantum dot (QD) in a two dimensional electron gas (2DEG) located 91nm below the surface of a GaAs-AlGaAs heterostructure. We deposit local top gates using standard electron beam lithography techniques in order to locally deplete the 2DEG and form the QDs. We operate between the states $(0,2)$ and $(1,1)$ where $\left(n_{L}, n_{R}\right)$ describes the state with $n_{L}\left(n_{R}\right)$ electrons in the left (right) QD. The $|S\rangle$ and $\left|T_{0}\right\rangle$ states, the logical subspace for the qubit, are isolated by applying an external magnetic field of $\mathbf{B}=700 \mathrm{mT}$ in the plane of the device such that the Zeeman splitting makes $T_{+}=|\uparrow \uparrow\rangle$, and $T_{-}=|\downarrow \downarrow\rangle$ energetically inaccessible. The exchange splitting, $J$, is a function of the difference in energy, $\epsilon$, between the levels of the left and right QDs. Pulsed DC electric fields rapidly change $\epsilon$, allowing us to switch $J$ on, which drives rotations around the z-axis. When $J$ is off the qubit precesses around the $\mathrm{x}$-axis due to a fixed $\Delta B_{z}$, which is stabilized to $\Delta B_{z} / 2 \pi=30 \mathrm{MHz}$ by operating the qubit as a feedback loop between interations of the experiment[12]. Dephasing of the qubit rotations reflects fluctuations in the magnitude of the two control axes, $J$ and $\Delta B$, caused by electrical noise and variation in the magnetic field gradient, respectively. The qubit is rapidly $(<50 \mathrm{~ns})$ initialized in $|S\rangle$ by exchanging an electron with the nearby Fermi sea of the leads of the QD in a region of $(0,2)$ where only $|S\rangle$ is accessible, and the qubit state is read out using standard Pauli blockade techniques, where $\epsilon$ is quickly tuned to the regime where $S$ occupies $(0,2)$ and $T_{0}$ occupies $(1,1)$, allowing the qubit state to be determined by the proximal charge sensor. The charge state of the qubit is rapidly read $(\sim 1 \mu s)$ using standard RF-techniques [13, 14] on an adjacent sensing QD.

In order to make use of the power of quantum information processing it is necessary to perform two qubit operations in which the state of one qubit is conditioned on the state of the other[15]. To investigate twoqubit operations we fabricate two adjacent $S$ - $T_{0}$ qubits such that they are capacitively coupled, but tunneling between them is suppressed (Fig. 1b). A charge sensing QD next to each qubit allows for simultaneous and independent projective measurement of each qubit (supplement). We use the electrostatic coupling between the qubits to generate the two-qubit operation[16]. When $J$ is nonzero, the $S$ and $T_{0}$ states have different charge configurations in the two QDs due to the Pauli exclusion principle (Fig. 1c). This charge difference, which is a function of $\epsilon$, causes the $|S\rangle$ and $\left|T_{0}\right\rangle$ states in one qubit to impose different electric fields on the other qubit. Since $J$ is a function of the electric field, the change imposed by the first qubit causes a shift in the precession frequency of the second qubit. In this way the state of the second qubit may be conditioned on the state of the 
first qubit. More precisely, when a single qubit evolves under exchange, there exists a state-dependent dipole moment, $\vec{d}$, between $|S\rangle$ and $\left|T_{0}\right\rangle$ resulting from their difference in charge occupation of the QDs. Therefore, when simultaneously evolving both qubits under exchange, they experience a capacitively mediated, dipoledipole coupling that can generate an entangled state. The two-qubit Hamiltonian is therefore given by:

$$
\mathscr{H}_{2-q u b i t}=\frac{\hbar}{2}\left(J_{1}\left(\sigma_{z} \otimes I\right)+J_{2}\left(I \otimes \sigma_{z}\right)+\frac{J_{12}}{2}\left(\left(\sigma_{z}-I\right) \otimes\left(\sigma_{z}-I\right)\right)+\Delta B_{z, 1}\left(\sigma_{x} \otimes I\right)+\Delta B_{z, 2}\left(I \otimes \sigma_{x}\right)\right)
$$

where $\sigma_{x, y, z}$ are the Pauli matrices, $I$ is the identity operator, $\Delta B_{z, i}$ and $J_{i}$ are the magnetic field gradients and the exchange splittings ( $\mathrm{i}=1,2$ for the two qubits), and $J_{12}$ is the two-qubit coupling, which is proportional to the product of the dipole moments in each qubit. For a two level system with constant tunnel coupling, the dipole moment scales as $\vec{d}_{i} \propto \frac{\partial J_{i}}{\partial \epsilon_{i}}$. Empirically, we find that for experimentally relevant values of $J_{i}, \frac{\partial J_{i}}{\partial \epsilon_{i}} \propto J_{i}(\epsilon)$, so that $J_{12} \propto J_{1} J_{2}$. As with the single qubit operations, this two-qubit operation requires only pulsed DC electric fields.

In principle, evolving both qubits under exchange produces an entangling gate. However, the time to produce this maximally entangled state exceeds the inhomogeneously broadened coherence times of each individual qubit, rendering this simple implementation of the two-qubit gate ineffective. To mitigate this we use a dynamically decoupled entangling sequence[17, 18](Fig. 1d). In this sequence, each qubit is prepared in $|S\rangle$ and is then rotated by $\frac{\pi}{2}$ around the $\mathrm{x}$-axis $\left(J_{i}=0, \Delta B_{z, i} / 2 \pi \approx 30 \mathrm{MHz}\right)$ to prepare a state in the $x-y$ plane. The two qubits are subsequently both evolved under a large exchange splitting $\left(J_{1} / 2 \pi \approx 280 \mathrm{MHZ}, J_{2} / 2 \pi \approx 320 \mathrm{MHz} \gg \Delta B_{z}\right)$ for a time $\frac{\tau}{2}$, during which the qubits begin to entangle and disentangle. A $\pi$-pulse around the $\mathrm{x}$-axis $\left(\Delta B_{z}\right)$ is then applied simultaneously to both qubits, after which the qubits are again allowed to exchange for a time $\frac{\tau}{2}$. This Hahn echo-like sequence[19] removes the dephasing effect of noise that is low frequency compared to $1 / \tau$, while the $\pi$-pulses preserve the sign of the two-qubit interaction. The resulting operation produces a CPHASE gate, which, in a basis of $\left\{|S S\rangle,\left|T_{0} S\right\rangle,\left|S T_{0}\right\rangle,\left|T_{0} T_{0}\right\rangle\right\}$, is an operation described by a matrix with $e^{-i \theta / 2}, 1,1, e^{-i \theta / 2}$ on the diagonals. For $\tau=\tau_{\text {ent }}=\frac{\pi}{2 J_{12}}(\theta=\pi)$ the resulting state is a maximally entangled generalized Bell state $\left|\Psi_{e n t}\right\rangle=e^{i \pi\left(I \otimes \sigma_{y}+\sigma_{y} \otimes I\right) / 8}\left|\Psi_{-}\right\rangle$, which differs from the Bell state $\left|\Psi_{-}\right\rangle=\frac{1}{\sqrt{2}}\left(|S S\rangle-\left|T_{0} T_{0}\right\rangle\right)$ by single qubit rotations.

In order to characterize our two-qubit gate and verify that we produce an entangled state we perform two-qubit state tomography and extract the density matrix and appropriate entanglement measures. The tomographic procedure is carefully calibrated with minimal assumptions in order to avoid adding spurious correlations to the data that may artificially increase the measured degree of entanglement (supplement). We choose the Pauli set representation of the density matrix[15, 20, 21], where we measure and plot the 16 two- 
qubit correlators $\langle i j\rangle=\left\langle\sigma_{i} \sigma_{j}\right\rangle$ where $\sigma_{i}$ are the Pauli matrices and $i, j \in\{I, X, Y, Z\}$. As a first measure of entanglement, we evaluate the concurrence[22] (Fig. 2a), $C(\rho)=\max \left\{0, \lambda_{4}-\lambda_{3}-\lambda_{2}-\lambda_{1}\right\}$ for different $\tau$, where $\rho$ is the experimentally measured density matrix, and $\lambda_{i}$ are the eigenvalues, sorted from largest to smallest, of the matrix $R=\sqrt{\sqrt{\rho}} \tilde{\rho} \sqrt{\rho}$, and $\tilde{\rho}=\left(\sigma_{y} \otimes \sigma_{y}\right) \rho^{*}\left(\sigma_{y} \otimes \sigma_{y}\right)$, and $\rho^{*}$ is the complex conjugate of $\rho$. A positive value of the concurrence is a necessary and sufficient condition for demonstration of entanglement [22]. For $\tau=140$ ns we extract a maximum concurrence of 0.44 .

While a positive value of the concurrence is a definitive proof of entanglement, it alone does not verify that the two-qubit operation produces the intended entangled state. In order to better characterize the generated quantum state, we evaluate another measure of entanglement, the Bell state fidelity, $F \equiv\left\langle\Psi_{\text {ent }}|\rho| \Psi_{\text {ent }}\right\rangle$. This may be interpreted as the probability of measuring our two-qubit state in desired $\left|\Psi_{\text {ent }}\right\rangle$. Additionally, for all non-entangled states one can show that $F \leq 0.5[23,24]$. In terms of the Pauli basis, the Bell state fidelity takes the simple form $F=\frac{1}{4} \vec{P}_{\text {ent }} \cdot \vec{P}_{\text {experiment }}$ where $\vec{P}_{\text {ent }}$ and $\vec{P}_{\text {experiment }}$ are the Pauli sets of a pure target Bell state and of the experimentally measured state, respectively. For our target state $\left|\Psi_{\text {ent }}\right\rangle$, the resulting Pauli set is given by $\langle X Z\rangle=\langle Z X\rangle=\langle Y Y\rangle=1$, with all other elements equal to zero (Fig 3a).

In an idealized, dephasing-free version of the experiment, as $\tau$ increases and the qubits entangle and disentangle, we expect the nonzero elements of the Pauli set for the resulting state to be

$$
\langle Y I\rangle=\langle I Y\rangle=\cos \left(J_{12} \tau\right),\langle X Z\rangle=\langle Z X\rangle=\sin \left(J_{12} \tau\right),\langle Y Y\rangle=1
$$

Dephasing due to electrical noise causes the amplitudes of the Pauli set to decay. However, the two-qubit Hamiltonian (equation(1)) includes rapid single-qubit rotations around the S- $T_{0}$ axis $\left(J_{1}, J_{2} \gg J_{12} / 2 \pi \approx 1 M H z\right)$ that change with $\tau$ due to imperfect pulse rise times in the experiment. These add additional single-qubit rotations around the $S$ - $T_{0}$ axis of each qubit, which are not accounted for in equation (2). We determine the angle of the single qubit rotations by performing a least-squares fit of the experimental data to modified form of equation (2) that accounts for these rotations and dephasing. The decays due to dephasing are fit by calculating $\rho(t)$ in the presence of noise on $J_{1}$ and $J_{2}$, which leads to decay of certain terms in the density matrix $[25,26]$. For the present case where $J_{12} \ll J_{1}, J_{2}$, we neglect the two-qubit dephasing, which is smaller than single-qubit dephasings by a factor of $\frac{J_{1}}{J_{12}}, \frac{J_{2}}{J_{12}} \approx 300$, and we extract a separate dephasing time for each individual qubit. We remove the single-qubit rotations numerically in order to simplify the presentation of the data (Fig. 3e). The extracted angles exhibit a smooth monotonic behavior which is consistent with their underlying origin (see supplemental information). 
In the absence of dephasing we would expect the Bell state fidelity to oscillate between 0.5 for an unentangled state and 1 for an entangled state as a function of $\tau$. This oscillation is caused by the phase accumulated by a CPHASE gate between the two qubits. However, the qubits dephase as the state becomes increasingly mixed, and this oscillation decays to 0.25 . Indeed, this behavior is observed (Fig. $2 \mathrm{~b}$ ): for very short $\tau$ there is very little dephasing present, and the qubits are not entangled. As $\tau$ increases the Bell state fidelity increases as the qubits entangle, reaching a maximum value of 0.72 at $\tau=140 \mathrm{~ns}$. As $\tau$ is increased further, we continue to see oscillations in the Bell state fidelity, though due to dephasing, they do not again rise above 0.5.

Fig. 2c shows these oscillations in Bell state fidelity as a function of $\tau$ for several different values of $J$ as $\epsilon$ is changed symmetrically in the two qubits. We see that as the value of $J$ increases in the two qubits, the time required to produce a maximally entangled state, $\tau_{e n t}$, decreases, but the maximum attainable fidelity is approximately constant. This is consistent with the theory that $J_{12} \propto \frac{\partial J_{1}}{\partial \epsilon_{1}} \cdot \frac{\partial J_{2}}{\partial \epsilon_{2}} \propto J_{1} \cdot J_{2}$.

To further understand the evolution of the quantum state, we focus on one value of $J$ and compare the measured Pauli set to that expected from single-qubit dephasing rates and $J_{12}$ (see supplemental information). Fig. 3a shows the Pauli set for the measured and expected quantum states for $\tau=40 \mathrm{~ns}$, which shows three large bars in the $\langle Y I\rangle,\langle I Y\rangle$, and $\langle Y Y\rangle$ components of the Pauli set. This is a nearly unentangled state. At $\tau=140 \mathrm{~ns}$, we see weight in the in the $\langle X Z\rangle,\langle Z X\rangle,\langle Y Y\rangle$ components of the Pauli set (Fig. 3b), and we extract a Bell state fidelity of 0.72 , which demonstrates the production of an entangled state. For $\tau=\tau_{\text {ent }}=\frac{\pi}{2 J_{12}}=160 \mathrm{~ns}$ (Fig. 3c) we see a similar state to $\tau=140 \mathrm{~ns}$, but with less weight in the single qubit components of the Pauli set. This state corresponds to the intended CPHASE of $\pi$, though it has a slightly lower fidelity than the state at $\tau=140 \mathrm{~ns}$ due to additional decoherence. Finally, at $\tau=\frac{\pi}{J_{12}}=320 \mathrm{~ns}$ (Fig. 3d), where we expect the state to be unentangled, we again see large weight in the $\langle Y I\rangle,\langle I Y\rangle$, and $\langle Y Y\rangle$ components of the Pauli set, though the bars are shorter than the Pauli set for $\tau=40 \mathrm{~ns}$, due to dephasing of the qubits. We plot the entire Pauli set as a function of time (Fig. 3e), which clearly shows the predicted oscillation (equation(2)) between $\langle Y I\rangle,\langle I Y\rangle$ and $\langle X Z\rangle,\langle Z X\rangle$, with decays due to decoherence.

The two-qubit gate that we have demonstrated is an important step toward establishing a scalable architecture for quantum information processing in $S-T_{0}$ qubits. State fidelity is lost to dephasing from

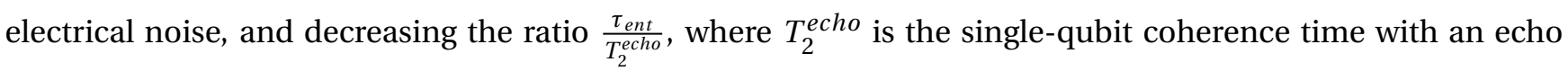
pulse, is therefore paramount to generating high-fidelity Bell states. Large improvements can be made by introducing an electrostatic coupler between the two qubits [27] in order to increase the two-qubit coupling $\left(J_{12}\right)$ and reduce $\tau_{e n t}$. We estimate that in the absence of other losses, if an electrostatic coupler were used, a Bell state with fidelity exceeding $90 \%$ could be produced. Additional improvements can be made 
by studying and mitigating the origins of charge noise to increase $T_{2}^{e c h o}$. This would allow future tests of complex quantum operations including quantum algorithms and quantum error correction. The generation of entangled also states opens the possibility of studying the complex dynamics of the nuclear environment, which is a fundamental, quantum, many body problem.

\section{Acknowledgments}

This work is supported through the NSA, "Precision Quantum Control and Error-Suppressing Quantum Firmware for Robust Quantum Computing” and IARPA “Multi-Qubit Coherent Operations (MQCO) Program.” This work was partially supported by the US Army Research Office under Contract Number W911NF-11-10068. This work was performed in part at the Center for Nanoscale Systems (CNS), a member of the National Nanotechnology Infrastructure Network (NNIN), which is supported by the National Science Foundation under NSF award no. ECS-0335765. CNS is part of Harvard University.

\section{Author Contributions}

V.U. prepared the crystal, M.D.S. fabricated the sample, M.D.S., O.E.D., H.B., S.P.H., A.Y. carried out the experiment, analyzed the data, and wrote the paper.

\section{Additional Information}

The authors declare no competing financial interests. Supplementary information accompanies this paper etc etc. Correspondence should be directed to AY at yacoby@physics.harvard.edu

\section{References}

1. D. Loss and D. P. DiVincenzo. Quantum computation with quantum dots. Phys. Rev. A, 57:120, 1998.

2. F. H. L. Koppens, C. Buizert, K. J. Tielrooij, I. T. Vink, K. C. Nowack, T. Meunier, L. P. Kouwenhoven, and L. M. K. Vandersypen. Driven coherent oscillations of a single electron spin in a quantum dot. Nature, 442:766, 2006. 
3. M. Pioro-Ladriere, T. Obata, Y. Tokura, Y. S. Shin, T. Kubo, K. Yoshida, T. Taniyama, and S. Tarucha. Electrically driven single electron spin resonance in a slanting Zeeman field. Nature Physics, 4:776, 2008.

4. K. C. Nowack, F. H. L. Koppens, Yu. V. Nazarov, and L. M. K. Vandersypen. Coherent control of a single electron spin with electric fields. Science, 318:1430, 2007.

5. M. Pioro-Ladriere, Y. Tokura, T. Kubo, and S. Tarucha. Micromagnets for coherent control of spin-charge qubit in lateral quantum dots. Appl. Phys. Lett., 90:024105, 2007.

6. H. O. H. Churchill, F. Kuemmeth, J. W. Harlow, A. J. Bestwick, E. I. Rashba, K. Flensberg, C. H. Stwertka, T. Taychatanapat, S. K. Watson, and C. M. Marcus. Relaxation and dephasing in a two-electron ${ }^{13} \mathbf{C}$ nanotube double quantum dot. Phys. Rev. Lett., 102:166802, Apr 2009.

7. S. Nadj-Perfe, S. M. Frolov, E. P. A. M. Bkkers, and L. P. Kouwenhoven. Spin-orbit qubit in a semiconductor nanowire. Nature, 468:1084, 2010.

8. J. Levy. Universal quantum computation with spin-1/2 pairs and Heisenberg exchange. Phys. Rev. Lett., 89:147902, 2002.

9. J. R. Petta, A. C. Johnson, J. M. Taylor, E. A. Laird, A. Yacoby, M. D. Lukin, C. M. Marcus, M. P. Hanson, and A. C. Gossard. Coherent manipulation of coupled electron spins in semiconductor quantum dots. Science, 309:2180, 2005.

10. J. M. Taylor, J. R. Petta, A. C. Johnson, A. Yacoby, C. M. Marcus, and M. D. Lukin. Relaxation, dephasing, and quantum control of electron spins in double quantum dots. Phys. Rev. B, 76:035315, 2007.

11. S. Foletti, H. Bluhm, D. Mahalu, V. Umansky, and A. Yacoby. Universal quantum control in two-electron spin quantum bits using dynamic nuclear polarization. Nature Physics, 5:903, 2009.

12. H. Bluhm, S. Foletti, D. Mahalu, V. Umansky, and A. Yacoby. Enhancing the coherence of a spin qubit by operating it as a feedback loop that controls its nuclear spin bath. Phys. Rev. Lett., 105:216803, 2010.

13. D. J. Reilly, C. M. Marcus, M. P. Hanson, and A. C. Gossard. Fast single-charge sensing with a RF quantum point contact. Appl. Phys. Lett., 91:162101, 2007.

14. C. Barthel, D. J. Reilly, C. M. Marcus, M. P. Hanson, and A. C. Gossard. Rapid single-shot measurement of a singlet-triplet qubit. Phys. Rev. Lett., 103:160503, 2009. 
15. M.A. Nielsen and I.L. Chuang. Quantum Computation and Quantum Information. Cambridge University Press, 2000.

16. J. M. Taylor, H.-A. Engel, W. Dür, A. Yacoby, C. M. Marcus, P. Zoller, and M. D. Lukin. Fault-tolerant architecture for quantum computation using electrically controlled semiconductor spins. Nature Physics, 1:177, 2005.

17. L. Viola, E. Knill, and S. Lloyd. Dynamical decoupling of open quantum systems. Phys. Rev. Lett., 82:2417, 1999.

18. D. Leibfried, B. Demarco, V. Meyer, D. Lucas, M. Barrett, J. Britton, W. M. Itano, B. Jelenkovic, C. Langer, T. Rosenband, and D. J. Wineland. Experimental demonstration of a robust, high-fidelity geometric two ion-qubit phase gate. Nature, 422:412, 2003.

19. E. L. Hahn. Spin echoes. Phys. Rev., 80:580, 1950.

20. Daniel F. V. James, Paul G. Kwiat, William J. Munro, and Andrew G. White. On the measurement of qubits. Phys. Rev. A, 64:052312, 2001.

21. J. M. Chow, L. DiCarlo, J. M. Gambetta, A. Nunnenkamp, Lev S. Bishop, L. Frunzio, M. H. Devoret, S. M. Girvin, and R. J. Schoelkopf. Detecting highly entangled states with a joint qubit readout. Phys. Rev. A, 81:062325, 2010.

22. Scott Hill and William K. Wootters. Entanglement of a pair of quantum bits. Phys. Rev. Lett., 78:5022-5025, Jun 1997.

23. C. A. Sackett, D. Kielpinski, B. E. King, C. Langer, V. Meyer, C. J. Myatt, M. Rowe, Q. A. Turchette, W. M. Itano, D. J. Wineland, and C. Monroe. Experimental entanglement of four particles. Nature, 404:6775, 2000.

24. Charles H. Bennet, Gilles Brassard, Sandu Popescu, Benjamin Schumacher, John A. Smolin, and William K. Wooters. Purification of noisy entanglement and faithful teleportation via noisy channels. Phys. Rev. Lett., 76:722, 1996.

25. Ting Yu and J.H. Eberly. Sudden death of entanglment: Classical noise effects. Opt. Commun, 264:393, 2006.

26. Lukasz Cywinski, Roman M. Lutchyn, Cody P. Nave, and S. Das Sarma. How to enhance dephasing time in superconducting qubits. Phys. Rev. B, 77:174509, 2008. 
27. Luka Trifunovic, Oliver Dial, Mircea Trif, James R. Wootton, Rediet Abebe, Amir Yacoby, and Daniel Loss. Long-distance spin-spin coupling via floating gates. Phys. Rev. X, 2:011006, Jan 2012. 


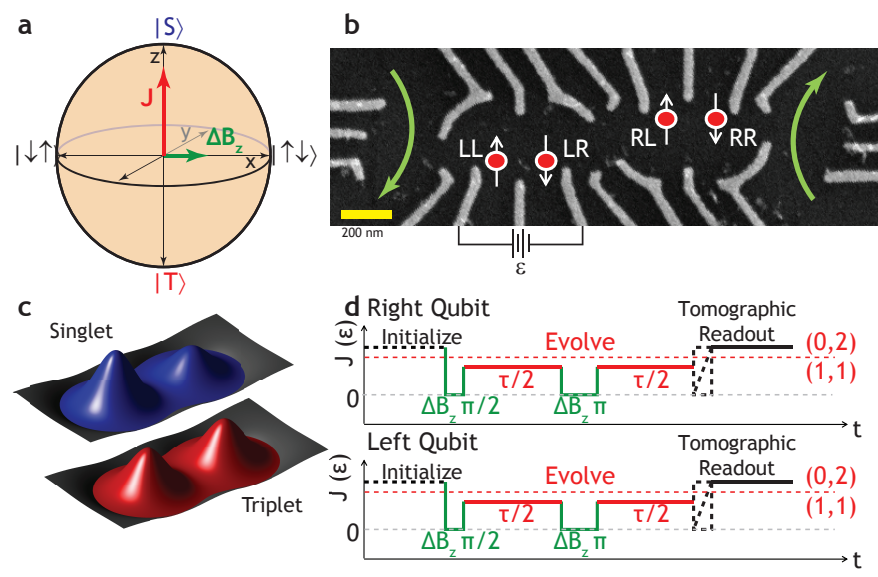

Figure 1 | Two-qubit coupling scheme. a, A Bloch sphere can be used to describe the states of the effective two-level system defined by the singlet and triplet states of the qubit, with the z-axis along the $S$ - $T_{0}$ axis and the $\mathrm{x}$-axis along the $|\uparrow \downarrow\rangle /|\downarrow \uparrow\rangle$ axes. $\mathbf{b}$, An SEM image of the top of the device used shows gates used to define the $S$ - $T_{0}$ qubits (white), dedicated ns control leads, the approximate locations of the electrons in the two qubits (red), and current paths for the sensing dots (green arrows). The left qubit uses the LR and LL electrons, while the right qubit uses the RL and RR electrons. c, A schematic of the electronic charge configurations for the $|S\rangle$ (blue) and the $\left|T_{0}\right\rangle$ (red) at non-zero $J$. This difference in charge configuration is the basis for the electrostatic coupling between the qubits d, The pulse sequence used to entangle the qubits: initialize each qubit in a $|S\rangle$, perform a $\pi / 2$ rotation around the $\mathrm{x}$-axis, allow the qubits to evolve under exchange for a time $\tau / 2$, perform a $\pi$-rotation around the $\mathrm{x}$-axis, thereby decoupling the qubits from the environment but not each-other, evolve under exchange for $\tau / 2$, and perform state tomography to determine the resulting density matrix (see supplemental information.) 

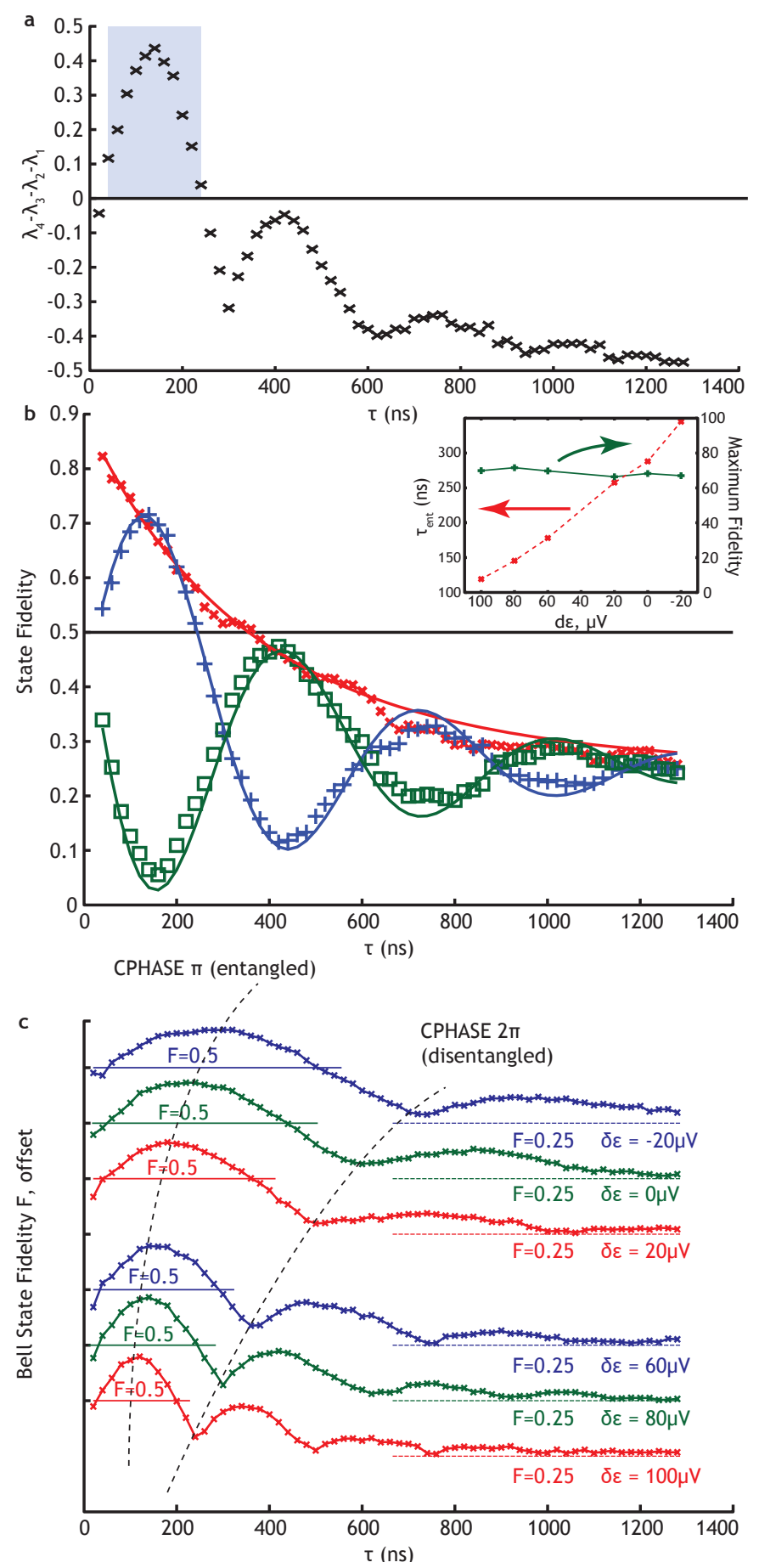

Figure 2 | Proof of entanglement: concurrence and state fidelity. a, A plot of the difference of the sorted eigenvalues of the matrix R, which for positive values is equal to the concurrence $c(\rho)$. States with a concurrence greater than zero (shaded region) are necessarily entangled. b. The fidelity with which the measured state approximates the target $\left|\Psi_{\text {ent }}\right\rangle$ (blue), and $e^{i \pi\left(\sigma_{y} \otimes I+I \otimes \sigma_{y}\right) / 4}\left|\Psi_{\text {ent }}\right\rangle$ (green), which differs from $\left|\Psi_{\text {ent }}\right\rangle$ by single qubit rotations and is the expected state for $\tau=3 \pi / 2 J_{12}$. The fidelity with which the measured state approximates a dephasing-free model of the entangling operation (red) shows smooth decay due to decoherence. The solid lines are fits to the data. Inset: The time to produce a maximally entangled state as a function of the change in $\epsilon$ (and therefore $J$ ) in the two qubits. As $J$ increases $\tau_{\text {ent }}$ decreases (red), but the maximum attainable fidelity (green) is approximately constant. Arrows indicate which y-axis is to be used. c, The Bell state fidelity as a function of time for different values of $J$ (offset) with guides to show where the fidelity exceeds $1 / 2$ for each curve. As $J$ increases in the two qubits the time to produce an entangled state, $\tau_{\text {ent }}$, decreases. 

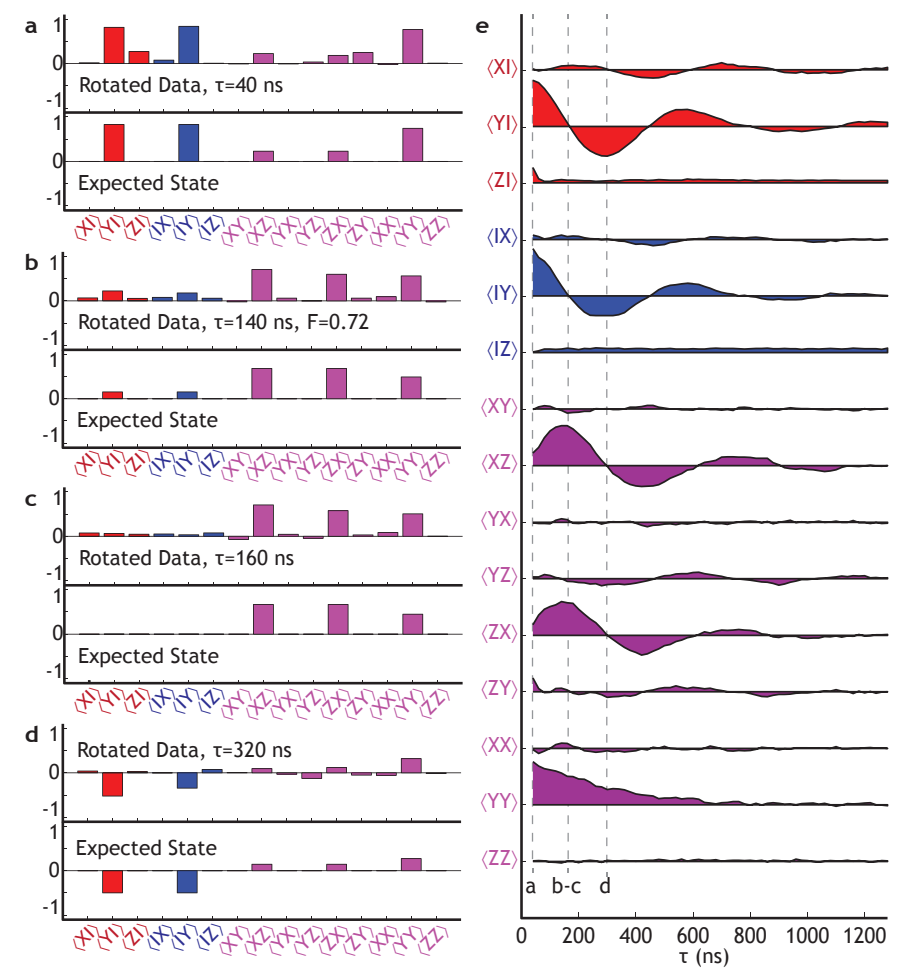

Figure 3 | Pauli set representation. a, The elements of the Pauli set for the measured density matrix and the state expected from the entangling and dephasing rates for small $\tau$ (unentangled). b, The Pauli set of the measured and expected states for $\tau=140 \mathrm{~ns}$, which produces a maximum Bell state fidelity of 0.72 . c. The Pauli set of the measured and expected states for $\tau=\tau_{\text {ent }}=160 \mathrm{~ns}$, which is a CPHASE of $\pi$ but does not the highest fidelity due to dephasing. d, The Pauli set for the measured and expected states for $\tau=320$ ns, which is an unentangled state. e, The full measured Pauli set as a function of $\tau$, which shows the expected behavior for a CPHASE gate. The the $y$-axes of adjacent elements in the Pauli set are offset by 1 . 


\title{
Supplementary Information for "Demonstration of Entanglement of Electrostatically Coupled Singlet-Triplet Qubits”
}

\author{
M. D. Shulman ${ }^{1}$, O. E. Dial ${ }^{1}$, S. P. Harvey ${ }^{1}$, H. Bluhm ${ }^{*}$, V. Umansky², and A. Yacoby ${ }^{1}$ \\ ${ }^{1}$ Department of Physics, Harvard University, Cambridge, MA, 02138, USA \\ ${ }^{2}$ Braun Center for Submicron Research, Department of Condensed Matter Physics, Weizmann Institute of Science, Rehovot 76100 Israel \\ ${ }^{*}$ Current Address: 2nd Institute of Physics C, RWTH Aachen University, 52074 Aachen, Germany
}

\section{Calibration of RF Sensor Response}

In order to quantitatively interpret sensor values for state tomography, it is important to precisely determine the RF sensor response that corresponds to a $|S\rangle$ or a $\left|T_{0}\right\rangle$ state. Because the state preparation is imperfect, it is in general difficult to accurately measure these values experimentally. To provide exact calibrations for $|S\rangle$ and $\left|T_{0}\right\rangle$, we exploit the fact that our sensor is capable of single shot readout. Histograms of sensor values for typical measurements yield a double-peaked curveone peak corresponds to $\left|T_{0}\right\rangle$ and one to $|S\rangle$ (Suppl. Fig 1a). In order to calibrate the sensor we first measure $T_{1}$ at the measurement point by preparing a state that is majority $\left|T_{0}\right\rangle$ (done with a $\pi$-pulse around the $\mathrm{x}$-axis) and fitting the sensor signal to a decaying exponential function of time elapsed before measurement (Suppl Fig. 1a). We note that the measured value of $T_{1}$ is a strong function of the power of the RF excitation used to read the conductance of the sensing QD. With prior knowledge of $T_{1}$, we use a procedure similar to that described in Barthel et. al (ref. 14) to optimize the measurement time given our signal to noise ratios and $T_{1}$. This process is repeated several times per day to check for drift. We recalibrate the sensor signals that correspond to $|S\rangle$ and $\left|T_{0}\right\rangle$ for each dataset (typically 10 minutes of acquisition time). For each set, we prepare a histogram of all observed sensor values. The presence of several reference measurements in each dataset guarantees that there will be a significant fraction of both $|S\rangle$ and $\left|T_{0}\right\rangle$. We then fit this double peaked curve to an analytic expression corresponding to a weighted sum of two Gaussians with some filling in due to $T_{1}$ decay during measurement (Suppl. Fig. 1b, purple line) as in ref. 14. From this, we extract the expected sensor distributions for $|S\rangle$ and $\left|T_{0}\right\rangle$ (blue and red lines in Suppl. Fig. $1 \mathrm{~b}$, respectively), as well as the fractions of $|S\rangle$ and $\left|T_{0}\right\rangle$ present. The centers of the two distributions correspond to the sensor signals that will be measured for pure $|S\rangle$ and pure $\left|T_{0}\right\rangle$, and using these values we can accurately scale the tomography data. We note that this procedure is insensitive to the percentages of $|S\rangle$ and $\left|T_{0}\right\rangle$. In our state tomography only expectation values are needed, so the single-shot capability of our readout is not necessary beyond this calibration. Nonetheless, we note that for the data presented, we measured readout fidelities of $97 \%$ and $98 \%$ for the left and right qubits, respectively.

\section{Readout Crosstalk}

For accurate state tomography it is important that the readout of the two qubits be independent. The two-qubit coupling relies on the fact that the $|S\rangle$ and $\left|T_{0}\right\rangle$ in one qubit electrostatically gate the other qubit, and reading out the two qubits
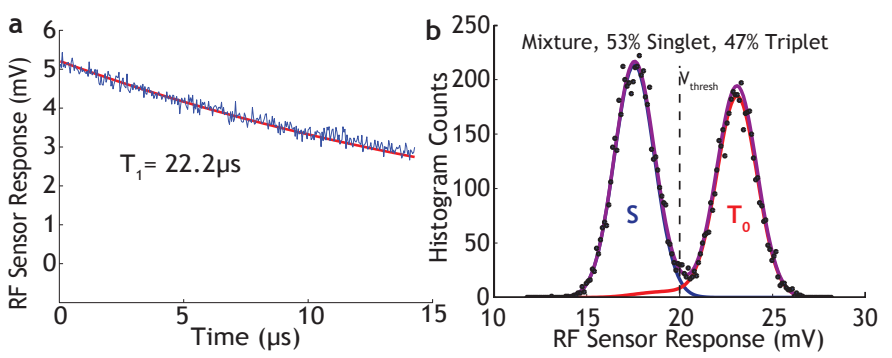

Supplementary Figure 1 | Singleshot Readout: a, The difference in sensor signal between $|S\rangle$ and $\left|T_{0}\right\rangle$ is fit to a decaying exponential to determine $T_{1}$, which is used in calibration of sensor values. $\mathbf{b}$, The histograms of a mixture of $|S\rangle$ and $\left|T_{0}\right\rangle$ states used to calibrate the sensor values. If we choose a threshold $V_{\text {thresh }}$ to distinguish between $|S\rangle$ and $\left|T_{0}\right\rangle$ we see a readout fidelity of $97 \%$. Purple: fit to noisy distribution including $T_{1}$ decay from $\left|T_{0}\right\rangle$ to $|S\rangle$. The deduced distribution for $|S\rangle$ (blue) is a Gaussian, while that for $\left|T_{0}\right\rangle$ (red) has a tail due to $T_{1}$ decay.

simultaneously leads to readout crosstalk; the left-qubit sensor value will be different for $|S\rangle\left|T_{0}\right\rangle$ and $|S\rangle|S\rangle$, and similarly for the right qubit (Suppl. Fig. 2d-e). We avoid this problem by reading out the qubits sequentially: while one qubit is read out the other qubit is "parked" deep inside of $(0,2)$ where the $(0,2)\left|T_{0}\right\rangle$ is lower in energy than the $(1,1)\left|T_{0}\right\rangle$ so that both the $|S\rangle$ and $\left|T_{0}\right\rangle$ occupy $(0,2)$ (Suppl. Fig. 2a-c). In this way each qubit is read out while the other qubit has the same charge distribution for the both qubit states. $T_{1}$ in this region (with the RF excitation off) is large, so no measurable degradation of the qubit being stored occurs.

\section{Calibration of State Tomography}

State tomography involves reading the projection of a qubit on to the three Cartesian axes of the Bloch sphere ${ }^{15}$. However, the charge sensor next to each qubit allows us to determine only the projection on to the $\mathrm{z}$-axis $\left(\mathrm{S}-T_{0}\right)$ of the qubit. Therefore, in order to perform state tomography, we apply rotations that map the $x$ and $y$ axes of the qubit to the $z$-axis ${ }^{11}$. The $y-$ axis component is mapped on to the minus $z$-axis by a $\pi / 2$ pulse around the $\mathrm{x}$-axis, which is driven by $\Delta B_{z}$, and the x-axis component is mapped on to the $\mathrm{z}$-axis by adiabatically turning on $J$, which maps the eigenstate $|\uparrow \downarrow\rangle(|\downarrow \uparrow\rangle)$ into $|S\rangle\left(\left|T_{0}\right\rangle\right)$ (Fig. 2a).

Extreme care must be taken when performing two-qubit state tomography, as errors in tomography can introduce spurious correlations which might inflate the measured degree of entanglement. However, in S- $T_{0}$ qubits, traditional methods for calibration are difficult to implement. The two control axes, $J$ and $\Delta B_{z}$, are not orthogonal, and precise 90 degree rotations are problematic due to the timing resolution available on our 


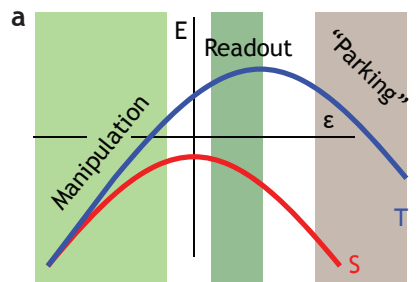

b

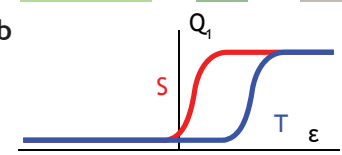

C

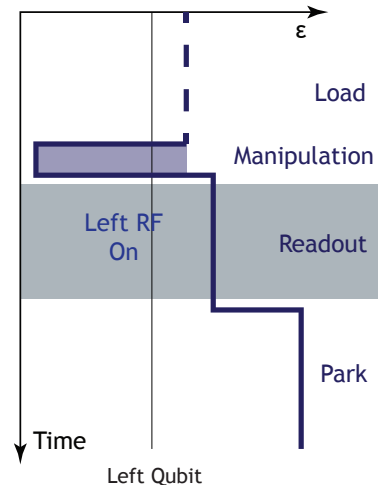

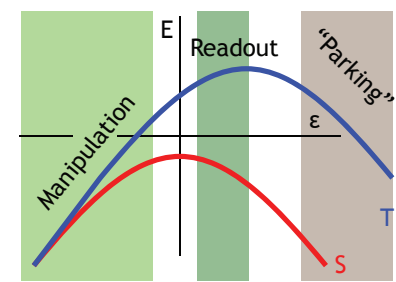

$\mathrm{Q}_{2}$
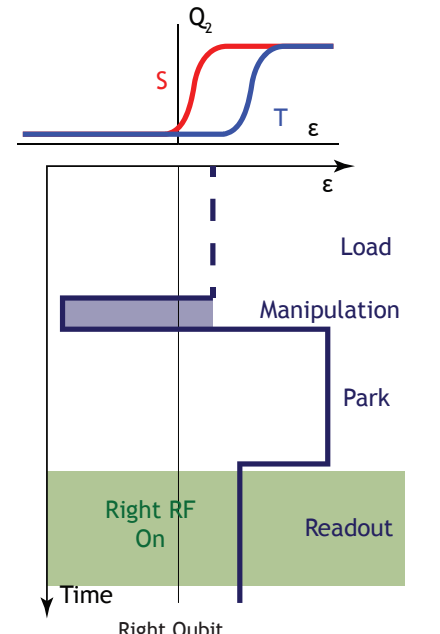

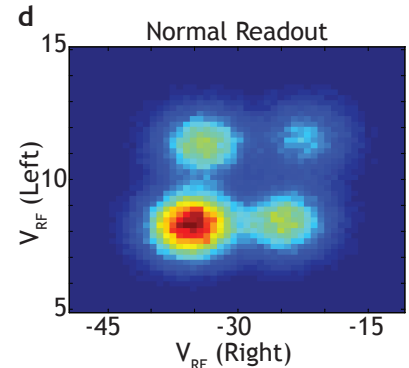

$\mathrm{V}_{\mathrm{RF}}$ (Right)

Reading Out a Singlet Peak shift due to crosstalk

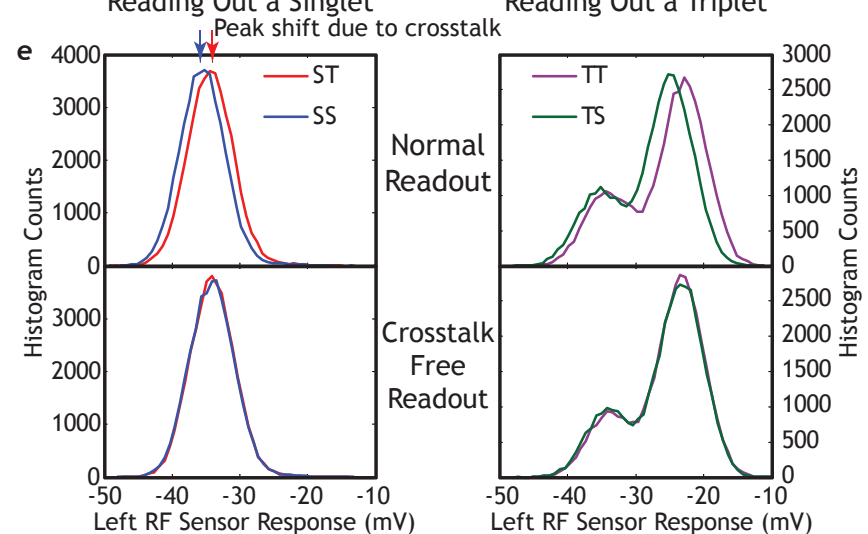

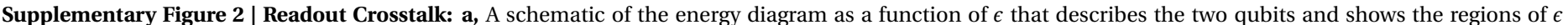

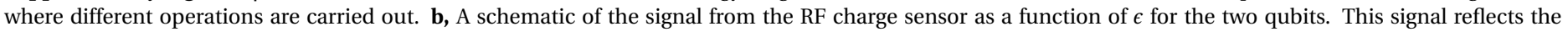

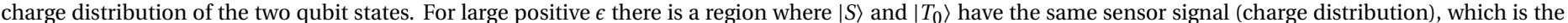

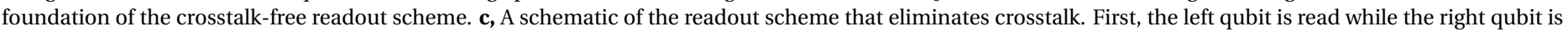

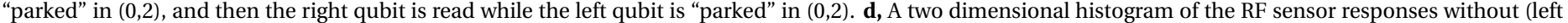

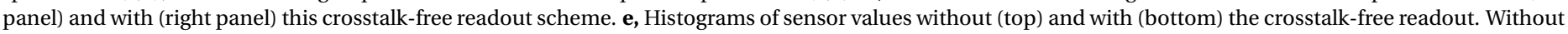
the crosstalk-free readout the sensor signal of one qubit depends on the state of the other qubit.

signal generators as well as pulse rise times. Pulse rise times in the experimental apparatus prevent instantaneous changes in $J$, which changes both the axis of rotation and the total angle rotated. These rise times also cause sudden changes in $J$ to become somewhat adiabatic. Furthermore, the effects of these pulse rise times depend both on the starting and ending point of the an individual pulse. Additionally, the two rotations involved in tomography of $S-T_{0}$ qubits are not easily tuned: the accuracies of both the $\mathrm{x}$ and $\mathrm{y}$-axis readouts are limited by the speed of the waveform generators $(\sim 1 \mathrm{~ns}$ in this work). Therefore, we perform careful measurements in order to determine the three axes on to which the qubit is projected, and apply a transformation in order to map these axes onto the traditional Cartesian axes. Dephasing during readout rotations is represented in this process as readout axes that are longer than the radius of Bloch sphere.

The procedure for determining the the tomographic axes makes minimal assumptions. We first calibrate the the $S-T_{0}$ readout using the singleshot histograms (see above). We then assume that any path of the state of the qubit around the Bloch sphere in a free-induction-decay experiment should smoothly dephase, i.e., there should not be oscillations in the amplitude of the Bloch vector. No assumptions are made about the axis or frequency of any rotation. If either the length or the angle of one of the tomography axes is incorrect, we expect to see "ripples" in the length of the measured Bloch vector as a function of evolution time(Fig. 3b-c). If we simultaneously consider many paths of evolution around the Bloch sphere, errors in the angles and lengths of the different readout axes become

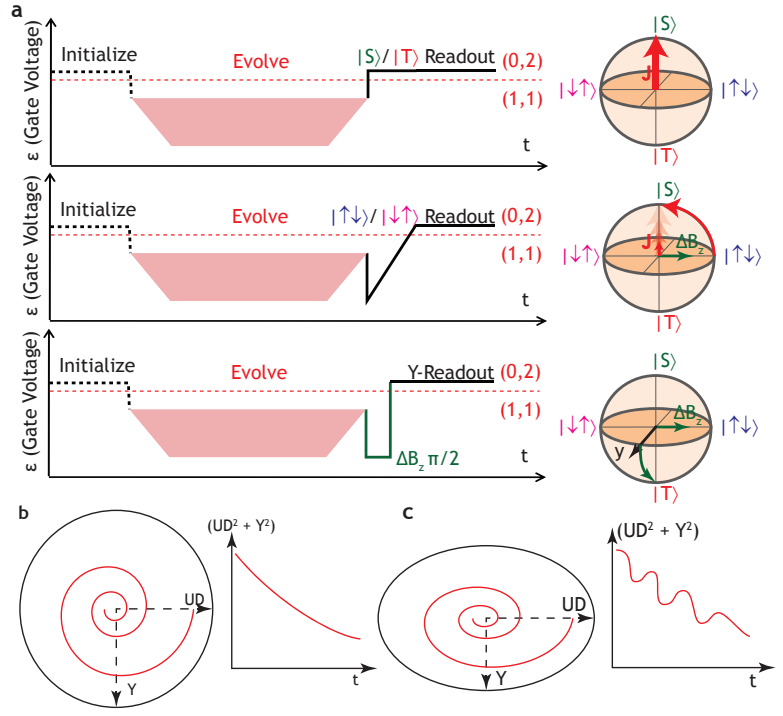

Supplementary Figure 3 | Calibration of state tomography: a, A schematic of how state tomography is performed in S- $T_{0}$ qubits ${ }^{11}$. The S- $T_{0}$ component is read out by charge sensing in a region where $|S\rangle$ occupies $(0,2)$ and $\left|T_{0}\right\rangle$ occupies $(1,1)$. The $\mathrm{x}(\mathrm{y})$ component is read adiabatically turning on $J$ (rotating by $\pi / 2$ around the $\mathrm{x}$-axis), followed by charge sensing. $\mathbf{b}$, A schematic of the length of the Bloch vector for perfect state tomography. c, A schematic of the "ripples" in the length of the Bloch vector if state tomography is flawed. The tomographic axes are determined by minimizing these ripples.

distinguishable due to varying phases, periods, and amplitudes of the ripples in the lengths of the measured vectors (Suppl. Fig. 4a). Therefore, to calibrate our axes, we gather data on 

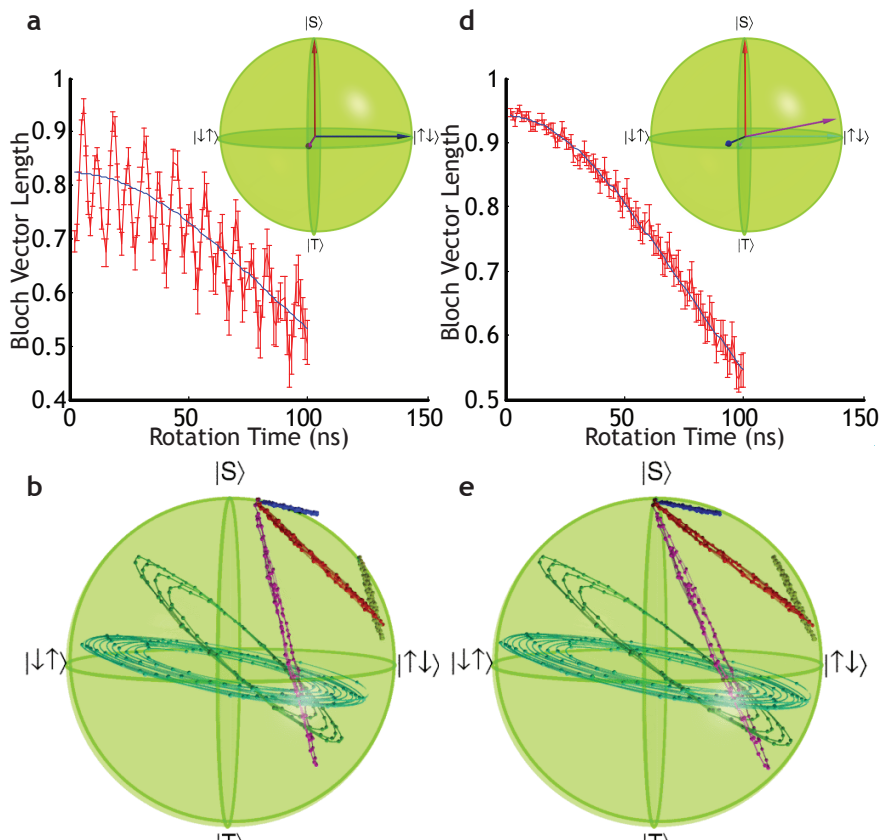

$|\mathrm{T}\rangle$

$|\mathrm{T}\rangle$

C

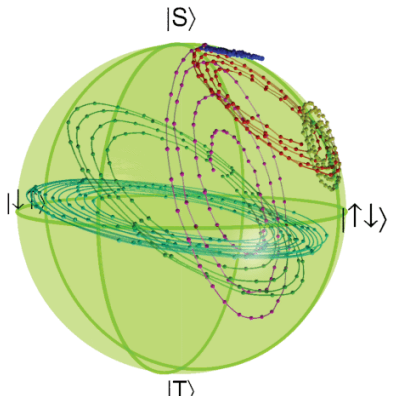

$|\mathrm{T}\rangle$

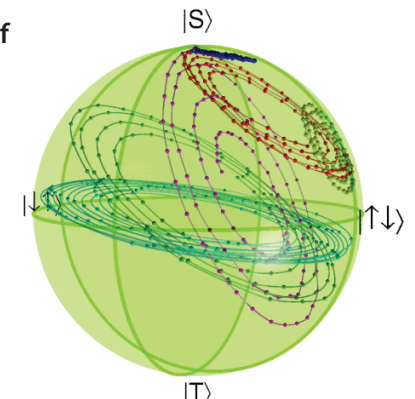

$|\mathrm{T}\rangle$

Supplementary Figure 4 | Calibrated vs uncalibrated state tomography: a, Data taken to calibrate the tomography shows ripples in the length of the Bloch vector if we assume that the tomography projects the quantum state on to Cartesian axes (inset). b-c, The paths around the Bloch sphere for the different evolutions that are used for tomography calibration. If the tomography is assumed to project on to the Cartesian axes there are points that lay outside the Bloch sphere, and the pure $|S\rangle$ states are not at the north pole, which is indicative of flawed state tomography. $\mathbf{d}$, The ripples in the length of the bloch vector are diminished (compared to panel a) if the axes deduced from state tomography (inset) are used. e-f, The paths around the Bloch sphere for the different evolutions that are used for state tomography. When the correct axes are used, all the points lie inside the Bloch sphere and the pure $|S\rangle$ are at the north pole.

many different evolutions around the Bloch sphere by evolving from many different starting points at many values of $\epsilon$ (Suppl. Fig. $4 \mathrm{~b}, \mathrm{c}, \mathrm{e}, \mathrm{f})$. We determine the axes on to which we project our state by finding the axes that minimize the amplitude of the ripples in the length of the Bloch vectors (Suppl. Fig. 4d). Based on our measurement procedure, we define the $S-T_{0}$ axis to lie along the z-axis. We allow the y-axis to lie anywhere on the Bloch sphere because a rotation around the $\mathrm{x}$-axis can suffer from over/under rotation as well as adiabaticity issues with switching $J$ on and off instantly. We constrain the x-axis to lie in the x-z-plane because the only expected error is due to adiabaticity turning $J$ on and off. The typical tomographic axes are shown in Suppl. Fig. 4d, and the signs of the errors are consistent with their origins. The variation from calibration to calibration is $\sim 1 \%$ on the axis lengths and angles.
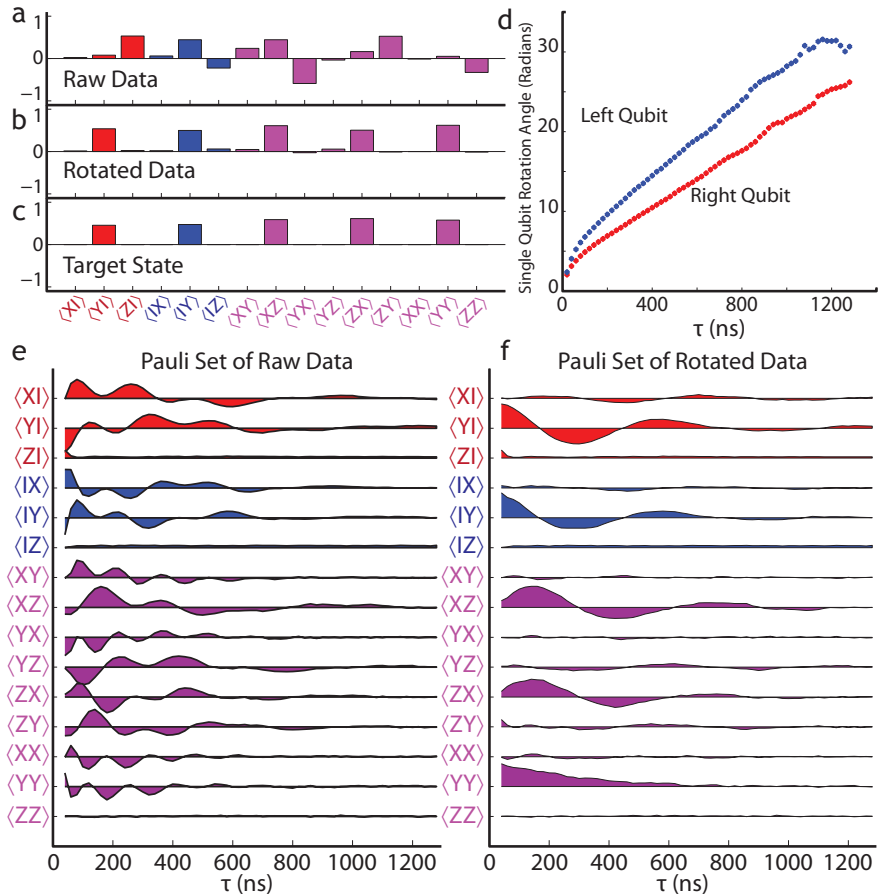

Supplementary Figure 5 | Single-qubit rotations: a, The Pauli set for $\tau=100 \mathrm{~ns}$ as measured is complicated by single qubit rotations. b, Numerically rotating each qubit around the $S-T_{0}$-axis simplifies presentation and analysis. c, The expected state for $\tau=100 \mathrm{~ns}$. d, The single qubit rotation angles for both qubits as a function of $\tau$ are smooth and monotonic functions. e-f The entire Pauli set as a function of $\tau$ for the raw and rotated data equation(1). The the y-axes of adjacent elements in the Pauli set are offset by 1.

\section{Determining Single Qubit Rotations}

During the entangling sequence the two qubits rotate very rapidly around the $S-T_{0}$ axis compared to the speed of the CPHASE gate $\left(J_{1} / 2 \pi \sim J_{2} / 2 \pi \sim 300 \mathrm{MHz}, J_{12} / 2 \pi \sim 1 \mathrm{MHz}\right)$. These single qubit rotations are not perfectly canceled out by the $\pi$ pulses in the dynamically decoupled sequence due to pulse distortions, consistent with pulse rise time effects at short times and capacitive coupling to RC-filtered DC gates at long times. Moreover, the angles by which the qubits are rotated change as a function of the evolution time $\tau$. In order to undo these rotations, we perform a least-square fit of the data to the expected form of the Pauli set (see equation(1) below), restricting the rotation to be around the S- $T_{0}$ axis because $J_{1}, J_{2} \gg \Delta B_{z}$. These angles are shown in Fig. $3 \mathrm{~b}$, and exhibit a smooth, monotonic behavior. The angles increase quickly for small $\tau$, which is consistent with pulse rise time effects, and display linear behavior for long $\tau$, which is consistent with long time RC filtering. For comparison, we plot the entire Pauli set for both the rotated and unrotated data in Suppl. Fig. 5 c-d. 


\section{Fit Form for a Dephased Bell State}

In order to fit the experimentally measured Pauli sets we calculate $\rho(t)$ in the presence of fluctuations on $J_{1}$, and $J_{2}$, and average over all these fluctuations ${ }^{25,26}$. For the present case where $J_{12} \ll J_{1}, J_{2}$, we neglect the two-qubit dephasing, which is smaller than single-qubit dephasings by a factor of $\frac{J_{1}}{J_{12}}, \frac{J_{2}}{J_{12}} \approx$ 300 . This yields the following non-zero elements of the Pauli set

$$
\begin{aligned}
\langle Y I\rangle & =e^{-\tau / T_{2,1}} \cos \left(J_{12} \tau\right) \\
\langle I Y\rangle & =e^{-\tau / T_{2,2}} \cos \left(J_{12} \tau\right) \\
\langle X Z\rangle & =e^{-\tau / T_{2,1}} \sin \left(J_{12} \tau\right) \\
\langle Z X\rangle & =e^{-\tau / T_{2,2}} \sin \left(J_{12} \tau\right) \\
\langle Y Y\rangle & =e^{-\tau / T_{2,1}} e^{-\tau / T_{2,2}}
\end{aligned}
$$

where $T_{2, i}$ are the single-qubit coherence times of the two qubits. From fits of the data to this form we extract for $\delta \epsilon=0$ : $T_{2,1}=420 \mathrm{~ns}, T_{2,2}=510 \mathrm{~ns}$, and $J_{12} / 2 \pi=0.87 \mathrm{MHz}$. Imperfect state preparation, in which the $\pi / 2$-pulse does not leave the state of the qubits in the $x-y$-plane causes mixing between terms, which is visible in the form of small amplitude oscillations in the $\langle X I\rangle,\langle I X\rangle$, and $\langle X X\rangle$ components of the Pauli set (Suppl. Fig. 5f). 